\title{
Advances and prospects in the field of waste management
}

\author{
Konstantinos Moustakas ${ }^{1} \cdot$ Maria Loizidou $^{1}$
}

Received: 29 August 2019 / Accepted: 23 September 2019 / Published online: 25 October 2019

(C) Springer-Verlag GmbH Germany, part of Springer Nature 2019

Without doubt, significant research is developed at world level in the area of waste management technologies and the current waste management status can improve substantially so as to contribute towards better life quality standards nowadays, especially in developing countries. This Special Issue of Environmental Science \& Pollution Research includes 28 environmental papers with emphasis on waste, which have been chosen based on quality and novelty standards by the Scientific Committee of the NAXOS 2018 6th International Conference on Sustainable Solid Waste Management (http://www.naxos2018.uest.gr) taken place in Naxos Island, Greece, from 13 to 16 June 2018 that was attended by more than 600 attendees from all continents.

After the previous five international conference events of this series, three in Athens in 2012, 2014, and 2017, one in Tinos Island, Greece in 2015, and one in Limassol, Cyprus, in 2016, the ambition of the NAXOS 2018 6th International Conference was to make a further step forward in the area of circular economy and waste management by presenting and sharing new aspects, experiences, and technological achievements at local, regional, and national level.

It is true that the series of our conferences on sustainable solid waste management continues to attract the attention of the international academic and scientific society and public and private sector. This is obvious by the substantial increase in the numbers of attendees of the conference, the number of origin countries of those traveling to Naxos, and the numerical data of contributions submitted and the number of full papers published in the NAXOS2018 conference proceedings.

Responsible Editor: Philippe Garrigues

Konstantinos Moustakas konmoust@central.ntua.gr

1 Unit of Environmental Science and Technology, School of Chemical Engineering, National Technical University of Athens, 9 Heroon Polytechniou Street, Zografou Campus 15780 Athens Greece
Significant work is undertaken by the organizing committee and especially the two authors of this editorial to maintain really high standards of quality in scientific work presented during the conference sessions and improve the visibility of the work presented at the conference, as demonstrated by the significant increase of the number of collaborating journals ( 8 for the case of NAXOS 2018 Conference) and the relevant impact factors, as well as the number of papers finally published in the special issues of the scientific journals. It is also our intention to provide the conference participants with highquality services and make them learn the Greek culture, traditions, hospitality, gastronomy, and landscape.

The NAXOS2018 Conference managed to attract the interest of the academia and business and notified them about the latest achievements in the field of solid waste management with special emphasis on municipal solid waste. Waste minimization and prevention, separation at source and recycling, composting and anaerobic digestion and generally biological waste treatment, and waste-to-energy also constituted highpriority conference topics. A new concept of the NAXOS2018 conference was the willingness to provide solutions for waste-related problems of islands and generally isolated areas. Emphasis was given to plastics, food waste, biomass and bio-based products, construction and demolition waste and other waste streams, and in general circular economy, including all key action areas and symbiosis networks.

The agenda of the NAXOS 2018 Conference was indeed dense and rich, covering a wide range of waste-related subjects presented in about 450 presentations fitted in 26 oral presentation sessions and the extensive 3-day poster session. The Cultural Center, former Ursuline School, was the conference venue. Naxos is well known for the diverse landscape of mountains and sea on top of its access to four surrounding smaller islands of Koufonisi, Irakelia, Schinousa, and Donousa, known as Small Cyclades. All five islands constitute touristic destinations catering for all ages and interests. Also, site visits took place on 16 June so that the participants become familiar with the PAVEtheWAySTE prototype systems targeting at source segregation and municipal solid waste 
treatment. These innovative systems were designed and constructed by the NTUA team and were installed in the Small Cyclades Islands and Ancient Olympia in the framework of the LIFE PAVEtheWAySTE project, in which the Municipality of Naxos and the small islands is the coordinating beneficiary, and NTUA has the main technical role. The scope is to provide local and regional authorities with the opportunity to improve the waste management status in isolated areas and, thus, enhance high resource recovery and circular economy. The special issue (NAXOS 2018) was prepared based on 28 articles of this conference.

The guest editors of this special issue have an extensive research background and expertise in waste management technologies, including biological and waste-to-energy technologies and biorefineries.

The 28 articles of this Special Issue involve different issues of sustainable waste management and involve different waste streams.

The 1st paper of the special issue handles with the use of fuzzy logic methodology and the establishment of a robust and novel interdisciplinary approach for systematically characterizing effects of uncertainties in social determinants of the management of municipal solid waste.

In the 2nd paper of the special issue, Branha et al. develop indicators to assess and communicate the performance of municipal waste management in a simple but comprehensive way-unlike indicators based on individual aspects such as recycling - and including aspects not well considered before, like waste prevention. The three dimensions of sustainability are covered, following an approach focused on dynamic progress towards sustainability instead of absolute achievements, contrary to the common static indicators, but complementing them. In the case studies tested, the framework proved to be reliable and of straightforward application in order to guide stakeholders.

The 3rd paper discusses a program for the environmental recovery of areas heavily degraded by the deposit of inert waste in Castilla y León (Spain). The program includes the restoration of illegal dumps bigger than $1000 \mathrm{~m}^{2}$, a total of 133 in the region, and the application of an alternative and legal system for managing construction and demolition waste. More than 2500 waste collection services were provided in 2017 managing $6000 \mathrm{t}$ of waste. Otherwise, this waste would probably have ended up in illegal dumps. These waste streams included discarded appliances (45\%), debris (33\%), and pruning $(22 \%)$. The management costs for these waste streams were $183.16 € / \mathrm{t}$ appliance, $25.53 € / \mathrm{t}$ debris, and $162.40 € / \mathrm{t}$ pruning.

Next, the 4th paper of the special issue addresses the landfill site's selection in an arid area (Babylon Governorate) in Iraq. Fifteen important criteria were selected to be taken into consideration. The multi-criteria decision-making (MCDM) methods were applied to ensure the suitable weight for each criterion. These methods include AHP and RSW. The change detection method was applied so as to determine the matching and non-matching pixels. The outcome revealed two potential landfill locations for each district in the governorate (ten sites). The areas of the selected sites are sufficient to receive the generated solid waste for the decade 2020-2030.

The 5th paper addresses the use of composts as soil amendments and fertilizers. The feasibility of using composts for these purposes was assessed at field level, in soft wheat crops and barley. The applied treatment consisted of two commercial composts (manure compost and sewage sludge compost) added to the soil either, alone or in combination with inorganic fertilizer, and a conventional mineral fertilization.

The 6th paper of the special issue deals with long-term research on the accumulation of nutrients and non-essential elements in fennel bulbs, onion bulbs, and tomato fruits following repeated soil fertilization using biowaste compost, mineral fertilizers, and a combination of them. Despite the compost-induced increase in soil available concentrations of a few elements, edible parts of crops grown on organic fertilized soils had invariably lower concentrations of micronutrients and non-essential elements than the ones from mineral fertilized or unfertilized soils. The use of biowaste compost can be thus regarded as an ideal fertilization practice, ensuring sustainability, high productivity, and, most importantly, high quality of the products.

The 7 th paper of the special issue suggests the manufacture of ceramic construction materials using exclusively industrial waste from several production processes as raw materials. From them, and using as a tool the $\mathrm{SiO}_{2}-\mathrm{Al}_{2} \mathrm{O}_{3}-\mathrm{CaO}$ diagram, two compositions were formulated: one that develops crystalline calcium phases (mixture $\mathrm{C}$ ) and another with a greater melting capacity (mixture D). The specimens have been sintered at three firing temperatures and have characterized microstructurally using SEM-EDX. In addition, the mineralogical composition was quantified by Rietveld refinement$\mathrm{XRD}$ and the distribution and pore size has been determined. These experiments have demonstrated that the ternary diagram $\mathrm{SiO}_{2}-\mathrm{Al}_{2} \mathrm{O}_{3}-\mathrm{CaO}$ is suitable for designing ceramic compositions starting from industrial waste.

The 8th paper of the special issue examines the relationship between inputs and outputs regarding energy amount and its corresponded economic value, in the greenhouse of vegetable crops on the island of Crete, Greece. Energy input-output analysis and data envelopment analysis were used to examine the major four crops cultivated on the island. The results also showed that the tomato-pepper case was the most efficient and profitable one. Based on the DEA approach, the average in scale efficiencies was as low as 0.80 . Consequently, the total input in euro could be significantly reduced without reducing the total output from its present level.

Preserving sewage sludge's $\mathrm{N}$ treating sludge with clay minerals was addressed in the 9th paper of the special issue. 
Nine clay minerals and additionally $\mathrm{Ca}(\mathrm{OH})_{2}$ were added to dewatered sewage sludge at $0-30 \%$ rates (wet weight basis). Then, the mixtures were equilibrated for 70 days and analyzed for certain properties. The results showed that the fecal indicators could not be detected in all cases of limed sludge and were considerably decreased compared with control only in the sludge treated with $30 \%$ of two bentonites, attapulgite, mixed clay of saponite-attapulgite, and zeolite.

The high quantities of waste generated by the marble industry, together with incorrect waste management, can cause environmental problems. It is for this reason that the authors of the 10th paper of the special issue have chosen to use the circular economy model to reduce the problem by incorporating the marble sludge generated in the ornamental rock industry as raw material in ceramic bricks.

The stabilization of sewage sludge by anaerobic digestion is sufficient to reduce the organic sludge content and to provide safely disposal of waste sludges, while producing energy via biogas according to the 11th paper of the special issue. Efficiency of anaerobic digestion that is represented as biogas/methane production and organic removal can be enhanced by pretreating the sludge prior to anaerobic digestion. This study compares the effects of microwave (MW), combined $\mathrm{H}_{2} \mathrm{O}_{2} / \mathrm{MW}$, and combined $\mathrm{H}_{2} \mathrm{O}_{2}$ /heat pre-treatments on the digestion efficiency and $\mathrm{CH}_{4}$ production potential of wastewater sludges. In addition, the $\mathrm{CH}_{4}$ production was calculated by using the modified Gompertz equation through the determination of the kinetic parameters. The pre-treatments applied to sludge samples improved the biodegradability of the organics through enhancing their solubility.

There is a need to set a challenge for exploration of lowcost, energy-efficient, and feasible solutions for the treatment and its future utilization with prime interests put on thermal conversion methods. The thermal conversion is considered as a promising alternative for effective management. Among three main thermochemical processes, it seems that gasification has many advantages. The aim of the 12th paper of the special issue is to present the gasification alternative as a sustainable management method taking into account the circular economy concept. Gaseous fuel production, solid adsorbent production, and $\mathrm{P}$ recovery potential are analyzed. The outcome of this paper shows that the lower heating value of the gas from gasification process is up to $5 \mathrm{MJ} / \mathrm{m}^{3}$ and it can be effectively utilized. The analysis confirmed that the solid fraction after the gasification process can be considered as a useful $\mathrm{P}$ source and perspective adsorbent materials.

The 13th paper of the special issue is part of a sustainable development approach wishing to promote viable thermal processing units for different agricultural waste streams, including tomato processing by-products, the potential of which such as biomass fuels is explored. Thermal process may, indeed, contribute to an environment friendly management and help tomato processing industries by developing new economic profitable circuits in an increasingly competitive context. For purposes of volume reduction on one hand and energy recovery on the other, pyrolysis was implemented in thermogravimetric conditions at different heating rates to these waste inputs. The suitability of the tomato waste as a potential bioenergy source was confirmed and the possible energy recovery from tomato wastes in the Tunisian context may contribute by approximately 16.3 thousand tons oil equivalent. Moreover, kinetic parameters were extracted for an accurate design of reactors.

The tire management system in Cyprus is addressed in the 14th paper of the special issue. Cyprus imported in 2015 almost $6 \%$ more new tires comparing to 2014, and those were equal to 835,142 pcs or $9638 \mathrm{t}$, while at the same year the total end-of-life tires (ELT) in the Island were 6629 t. The existing ELT management system consists from three private sectors and had the ability to collect on 2016 almost $7201 \mathrm{t}$. ELT in Cyprus were used as alternative fuel from the cement industry as well as to produce granules. They are also used to develop artificial surfaces, such as tennis court, or to pyrolyze them for energy recovery. The total revenue from the new tires that are entering in the Island exceeded 33 million $€$. On the basis of the existing management system using SWOT analysis, the main weaknesses were the gap in the national legislation related with specific collected target, while the main threats include the bureaucracy as well as the technical and financial viability.

In the 15th paper of the special issue by Chrysargyris et al., olive-stone waste was used for peat replacement in growing media for Brassica seedling production. Olive-stone waste mixed with peat affected growing media properties and provided minerals. High ratio of olive-stone waste decreased plant growth, chlorophylls, and leaf stomatal conductance but increased $\mathrm{K}, \mathrm{Ca}$, phenolics, and antioxidative enzyme metabolism, indicating that up to $20 \%$ of olive-stone waste can replace peat for broccoli, cabbage, and cauliflower seedling production.

Vakalis et al. presented the idea of the "COFFEE BIN" in the 16th paper of the special issue. This paper merged the separate collection of spent coffee grounds, i.e., the "COFFEE BIN", with thermal valorization applications where the optimal process was found to be torrefaction. This scheme produced very final high solid yields, i.e., from 78 to $83 \%$, with heating values of about $25 \mathrm{MJ} / \mathrm{kg}$. Higher temperature pyrolysis missed to maintain the advantage of increased returned solid mass yields.

Recent advances in the application of microfluidic tools for lipid production and modification were reviewed in the 17th paper of the special issue. This review has excavated the application of microfluidic tools on the discovery, culturing, harvesting, separating, and monitoring of lipid-producing microorganisms. In summary, because microfluidic tools are good at regulating and controlling the extremely small amount of fluid 
with characteristics of superlatively fast and efficient mass and heat transfer, it has been considered as a novel tool for lipid production and modification, which have great potential as an efficient tool for a large range of industrial applications. Furthermore, the explorative of lipid resources regarded as an alternative renewable energy source is a link to discover the novel energy resource.

The authors of the 18th paper of the special issue demonstrated that inositol as an effective enhancer significantly promoted the accumulation of lipids and unsaturated fatty acids (UFAs) in the fermentation of Schizochytrium sp. SR21. They found the best culture conditions and confirmed the key enzymes and key metabolic pathways through metabolomics studies. Metabolomics showed that inositol promoted lipid synthesis in SR21 and significantly increased the relative proportion of UFAs through affecting the citrate cycle and saturated fatty acid (SFA) and UFA metabolic pathways. Thus, inositol possesses a potential application in improving lipid accumulation and production by oleaginous microorganisms.

In the 19th paper of the special issue, the effect of $\mathrm{pH}$ on the production of volatile fatty acids in mesophilic semicontinuous fermenters using food waste from a canteen of the university and organic fraction of municipal solid waste from a mechanical-biological treatment plant was studied. Under acidic conditions ( $\mathrm{pH}$ 6.0), an effluent with VFA concentration up to $11.5 \mathrm{~g} \mathrm{~L}^{-1}$ was obtained for both substrates. However, the VFA profile was more enriched in C3 and C5 acids when using organic fraction of municipal solid waste. At pH 9.5-10, an enhanced solubilization of organic matter was registered and, for the case of food waste, volatile fatty acids production was also improved, being acetic acid the predominant product.

Crude glycerol and soybean cake were used as sole feedstocks producing 1,3-propanediol with the use of two bacterial strains of Citrobacter freundii in the 20th paper of the special issue. Soybean cake was converted into a nutrient-rich hydrolysate by crude enzymes produced via solid-state fermentation. The cultivation of $C$. freundii VK-19 in fed-batch bioreactor cultures using crude glycerol and soybean cake hydrolysates led to 1,3-propanediol concentration of $47.4 \mathrm{~g} / \mathrm{L}$ with yield and productivity of $0.49 \mathrm{~g} / \mathrm{g}$ and $1.01 \mathrm{~g} / \mathrm{L} / \mathrm{h}$, respectively. The effect of 1,3-propanediol, metabolic by-products, and $\mathrm{Na}$ and $\mathrm{K}$ salts on bacterial growth was assessed. The results showed that $\mathrm{K}$ salts first help bacterial growth, whereas $\mathrm{Na}$ salts lead to substantial inhibition to the growth of bacteria.

The 21 st article of the special issue deals with the manufacturing process of the polypropylene, where more than 60 grades of polypropylene are produced step by step with changes in the process variables. Then, those changes generate different mixtures of resins with intermediate properties (transitions) that usually are considered low price products or in some cases residues. It is proposed to analyze the important influence on the environment, when these transitions are considered as waste. In the paper, an environmental analysis was made by using a waste reduction algorithm to estimate the potential impact of a polypropylene production process over the environment, applying grade transitions strategies and then using this information to improve the transition times to reduce the plastic waste. The most interesting characteristic of this article is the fact that all the work is based on real data from 300 tons/day plant in Colombia.

Non-thermal plasma and ultrasound-assisted open lactic acid fermentation of distillery stillage achieves two simultaneous goals: first, a significant microbial reduction and secondly high lactic acid productivity and yield. By tailoring treatment parameters, the authors of the 22nd article of the special issue demonstrated that the highest lactic acid productivity of $1.21 \mathrm{~g} / \mathrm{Lh}$ and yield of $0.82 \mathrm{~g} / \mathrm{g}$ can be achieved using plasma treatment. Ultrasound treatment of $10 \mathrm{~min}$ provided productivity of $1.02 \mathrm{~g} / \mathrm{Lh}$ and lactic acid yield of $0.69 \mathrm{~g} / \mathrm{g}$. Benchmarked against closed lactic acid fermentation $20 \%$ better revalorization of stillage by plasma treatment and open lactic acid fermentation was achieved. At the same time, an excellent L (+) lactic acid stereoselectivity of $95.5 \%$ was attained.

The 23rd paper of the special issue by Papanikola et al. discussed the production of alternative fuel to be used for making cement from a food residue biomass product, gained from separated at source food waste of household origin. The food residue biomass is obtained after drying and shredding the fermentable fraction of food waste collected door-to-door in a Greek municipality. The main properties were then determined using well-established standards. The food residue biomass was evaluated as potential fuel in terms of environmental impacts and technical feasibility. The food residue biomass was classified as a non-dangerous waste and a suitable candidate as secondary fuel for the producing cement, given its low humidity and ash content along with its high calorific value.

More than a third of the total waste generated in the European Union corresponds to construction and demolition waste. Its heterogeneity, in terms of composition, makes its application difficult. For instance, the gypsum content limits its use as sub-base or base layer in road construction. Hence, the 24th article of this special issue evaluates the effect of gypsum, at different levels, of recycled aggregates on mechanical properties, leaching behavior, and dimensional changes at long term.

The 25th paper of the special issue proposes the manufacture of ceramic construction materials using raw materials which are just industrial waste deriving from different production processes. The $\mathrm{SiO}_{2}-\mathrm{Al}_{2} \mathrm{O}_{3}-\mathrm{CaO}$ equilibrium phase diagram has been used in order to formulate two material compositions with the objective of obtaining ceramics with chemical composition in the same area as a traditional clay-based material, but with two different technological behaviors: refractoriness due to calcium phase's 
development and densification due to a greater melting capacity. The conclusion is that extending the use of phase diagrams as a design tool is still little explored for wastebased ceramics, while those that have been mainly used for studying Mg-based phases can have an important contribution to sustainability in the construction sector.

Exploitation of mineral resources can generate large waste quantities that can lead to the generation of contaminated water, such as acid mine drainage that is characterized by low $\mathrm{pH}$ and high concentrations of metals and sulfates. The 26th article of the special issue evaluated the leaching potential of contaminants from solids after over 1-year treatment of Ferich acid mine drainage. Three post-treatment residues (WA50 - wood ash 50\%, wood chips 50\%; PBR-passive biochemical reactor, $30 \%$ inorganic materials, $70 \%$ organic substrate; and C50 - calcite 50\%, wood chips 50\%) were sampled from a laboratory Fe-acid mine drainage passive treatment system. Physicochemical and mineralogical characterization, as well as static leaching tests, was then performed.

Contaminated port sediments represent an important management problem that also concerns their remediation. Port dredging activities generate huge volumes of contaminated sediments every year that have to be handled effectively due to their inorganic and organic polluting load. The goal of the 27 th paper of the special issue was to characterize the fungal communities of port sediments in Genoa and present an innovative mycoremediation protocol to assess the capability of indigenous fungal strains in the remediation from heavy metals. The results show efficient $\mathrm{Cu}$ and $\mathrm{Zn}$ bioremoval from sediments.

The last paper of this special issue presents the economic and environmental benefits of treating electronic waste for the member states of the Gulf Cooperation Council (GCC). The results of this paper would constitute an essential step for future studies for electronic waste in the region. A novel initiative to develop a unified free zone to recycle the electronic waste in GCC member states that would meet the standards and regulations at local, regional, and international level is proposed.

Acknowledgements We would like to express our thanks to the EditorIn-Chief of Environmental Science \& Pollution Research, Dr. Philippe Garrigues, for trusting us in order to publish this Special Issue on advances and prospects in the field of waste management, as well as the LIFE+ Programme of the European Commission for supporting financially the LIFE PAVEtheWAySTE project.

Publisher's note Springer Nature remains neutral with regard to jurisdictional claims in published maps and institutional affiliations.

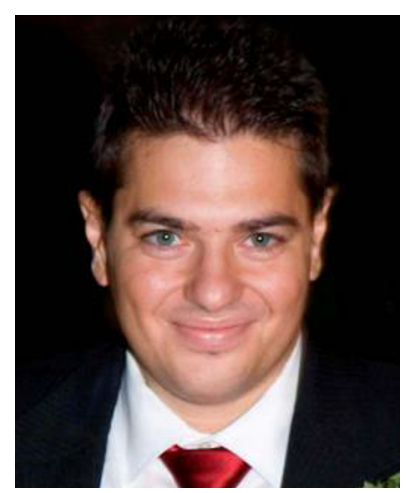

Konstantinos Moustakas is Chemist, with $\mathrm{PhD}$ in waste management. He works as Laboratory-Teaching Staff in the Unit of Environmental Science \& Technology (www.uest.gr) of the School of Chemical Engineering of the National Technical University of Athens. He has participated in more than 60 projects in several countries. He has long teaching experience and considerable international experience as project manager and senior researcher. He has cooperated with the European Commission directly, as he acted as TAIEX expert (institutional building) in many countries. He has long training activity in the field of environment in different countries and has been Collaborating Teaching Staff for the Hellenic Open University for five years (Subject: Solid Waste Management). He has published more than 250 articles in conferences and journals, and he has served as Guest Editor in more than 15 Special Issues, while he has had the key role in the organization of a large number of international conferences. Indicatively, he is the Head of the Organising Committee and key member of the Scientific Committee for the successful series of conferences on Sustainable Solid Waste Management.

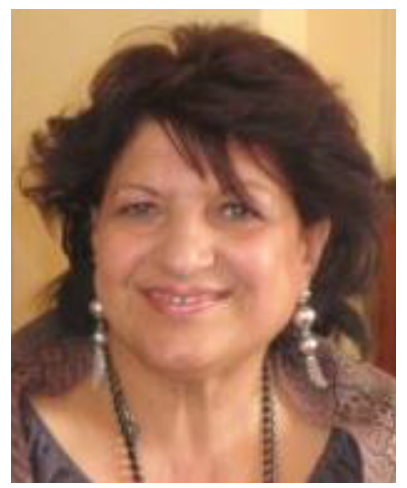

Maria Loizidou obtained her $\mathrm{PhD}$ in chemical engineering focusing on the field of environmental protection at the University of London. She followed an academic career being Professor at the National Technical University of Athens in the School of Chemical Engineering and Head of the Unit of Environmental Science \& Technology (www.uest.gr).

Her efforts are continuous in the field of the environmental protection and human health, enhancing education, research technology, and innovation. She has been scientific responsible for more than 175 environmental projects supporting competent authorities, municipalities, and others (http:// www.uest.gr/index.php/projects). She has more than 600 publications in international scientific journals (http://www.uest.gr/index.php/ publications) and conferences and more than 7500 citations.

Prof. Loizidou is the Head of the Scientific Committee of this series of conferences on Sustainable Waste Management bringing together academics, private and public sector, municipalities and regions, and professionals from all continents.

Prof. Loizidou won the first Green AWARD with the LIFE SOLBRINE project that was voted as the best LIFE ENVIRONMENT project for the period 1992-2017 among more than 4000 LIFE projects. 